THE ASTROPHYSICAL JOURNAL, 516:187-194, 1999 May 1

(c) 1999. The American Astronomical Society. All rights reserved. Printed in U.S.A.

\title{
THE ORIGIN OF THE NARROW-LINE REGION OF MARKARIAN 3: AN OVERPRESSURED JET COCOON ${ }^{1}$
}

\author{
A. CAPETTI \\ Osservatorio Astronomico di Torino Strada Osservatorio 20, 10025 Torino, Italy; capetti@to.astro.it \\ D. J. Axon $^{2}$ \\ Division of Physical Sciences, University of Hertfordshire, College Lane, Hatfield, Herts AL10 9AB, UK; axon@stsci.edu \\ F. D. MACCHETTO ${ }^{2}$ \\ Space Telescope Science Institute, 3700 San Martin Drive, Baltimore, MD 21218; macchetto@stsci.edu
}

A. MARCONI

Osservatorio Astrofisico di Arcetri, Largo E. Fermi 5, 50125, Firenze, Italy; marconi@arcetri.astro.it

AND

C. WINGE ${ }^{3}$

Instituto de Física, Universidade Federal do Rio Grande do Sul, Avenue Bento Gonçalves 9500, C.P. 15051, CEP 91501-950, Porto Alegre, RS, Brazil; winge@if.ufrgs.br

Received 1998 August 12; accepted 1998 December 8

\begin{abstract}
We have obtained Hubble Space Telescope (HST) Faint Object Camera (FOC) f/48 long-slit optical spectroscopy of the inner $2^{\prime \prime}$ of the narrow-line region (NLR) of the Seyfert 2 galaxy Mrk 3 with a spatial resolution of 0.06 . Spectra were taken in six locations with the slit approximately perpendicular to the radio axis. In the region cospatial with the radio jet, where the brightest emission line knots are located, the velocity field is highly perturbed, showing two velocity systems separated by as much as $1700 \mathrm{~km} \mathrm{~s}^{-1}$. In several locations the split lines form almost complete velocity ellipsoids, implying that we are seeing an expanding shell of gas. The diameter of this shell $(\sim 200 \mathrm{pc})$ is much larger than the width of the radio jet $(d<15 \mathrm{pc})$. We interpret this to be the consequence of the rapid expansion of a cocoon of hot gas, shocked and heated by the radio-emitting outflow, which compresses and accelerates the ambient gas. The cocoon mediates the energy exchange between jets and the line-emitting gas. The gas motions within the NLR of Mrk 3 are therefore clearly dominated by the interaction between the jets and the interstellar medium; the NLR itself is essentially a cylindrical shell expanding supersonically. With its current size of $200 \mathrm{pc}$, the cocoon has expanded to several disk scale heights. Because of the external gas density stratification, the hot gas located above the plane of the disk blows out into the halo, puncturing the bubble and fracturing the velocity ellipsoids. The system is effectively momentum driven. From the size and velocity of the expanding region, we derive an upper limit to the age of the radio source of $\lesssim 1.5 \times 10^{5} \mathrm{yr}$, and a lower limit for the jet power of $\gtrsim 2 \times 10^{42} \mathrm{ergs} \mathrm{s}^{-1}$, required to

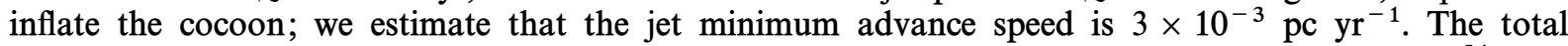
kinetic energy of the high-velocity gas associated with the radio jet can be estimated as $\sim 6 \times 10^{54}$ ergs, comparable to the total energy carried by the jet over its lifetime; this quantitatively supports the idea that the NLR gas is accelerated by the jet. Radio outflows are associated with at least $50 \%$ of Seyfert galaxies with typical sizes smaller than a few kpc. If the advance speed of Mrk 3 is representative of the Seyfert population, then these sources must also be short lived and are probably recurrent. Evidence that this is indeed the case is provided by the fact that the expansion timescale derived for NGC 1068 is comparable to that seen in Mrk 3. The jet kinetic luminosity of Mrk 3 is between 2 and 3 orders of magnitude smaller than that derived for radio-loud AGNs with similar emission-line luminosity. On the other hand, the fraction of jet power dissipated in radio emission is similar. We speculate that the main distinction between radio-quiet and radio-loud active galactic nuclei can be ascribed to a difference in jet power rather than to a different efficiency in synchrotron emission production.
\end{abstract}

Subject headings: galaxies: active - galaxies: individual (Markarian 3) - galaxies: jets galaxies: Seyfert

\section{INTRODUCTION}

During the last two decades, ground-based studies of Seyfert galaxies have firmly established that a close relationship exists between radio and line emission. In particular,

\footnotetext{
${ }^{1}$ Based on observations with the NASA/ESA Hubble Space Telescope, obtained at the Space Telescope Science Institute, which is operated by AURA, Inc., under NASA contract NAS 5-26555 and STScI grant GO3594.01-91A.

${ }^{2}$ Affiliated with the Astrophysics Division, Space Science Department, ESA.

${ }^{3}$ CNPq Fellowship, Brazil.
}

they have shown that the narrow-line region (NLR) is inevitably cospatial with the radio emission (e.g., Wilson \& Ulvestad 1983; Haniff, Wilson, \& Ward 1988). Furthermore, galaxies harboring a linear radio source have unusually wide line profiles (Whittle 1985) and display a correspondence between the location of the high-velocity NLR gas and the location of the radio ejecta (e.g., Whittle et al. 1988; Baldwin, Wilson, \& Whittle 1987; Pedlar et al. 1989). This spatial and kinematical association has been interpreted as resulting from the compression and acceleration of the interstellar gas induced by the expansion of the 


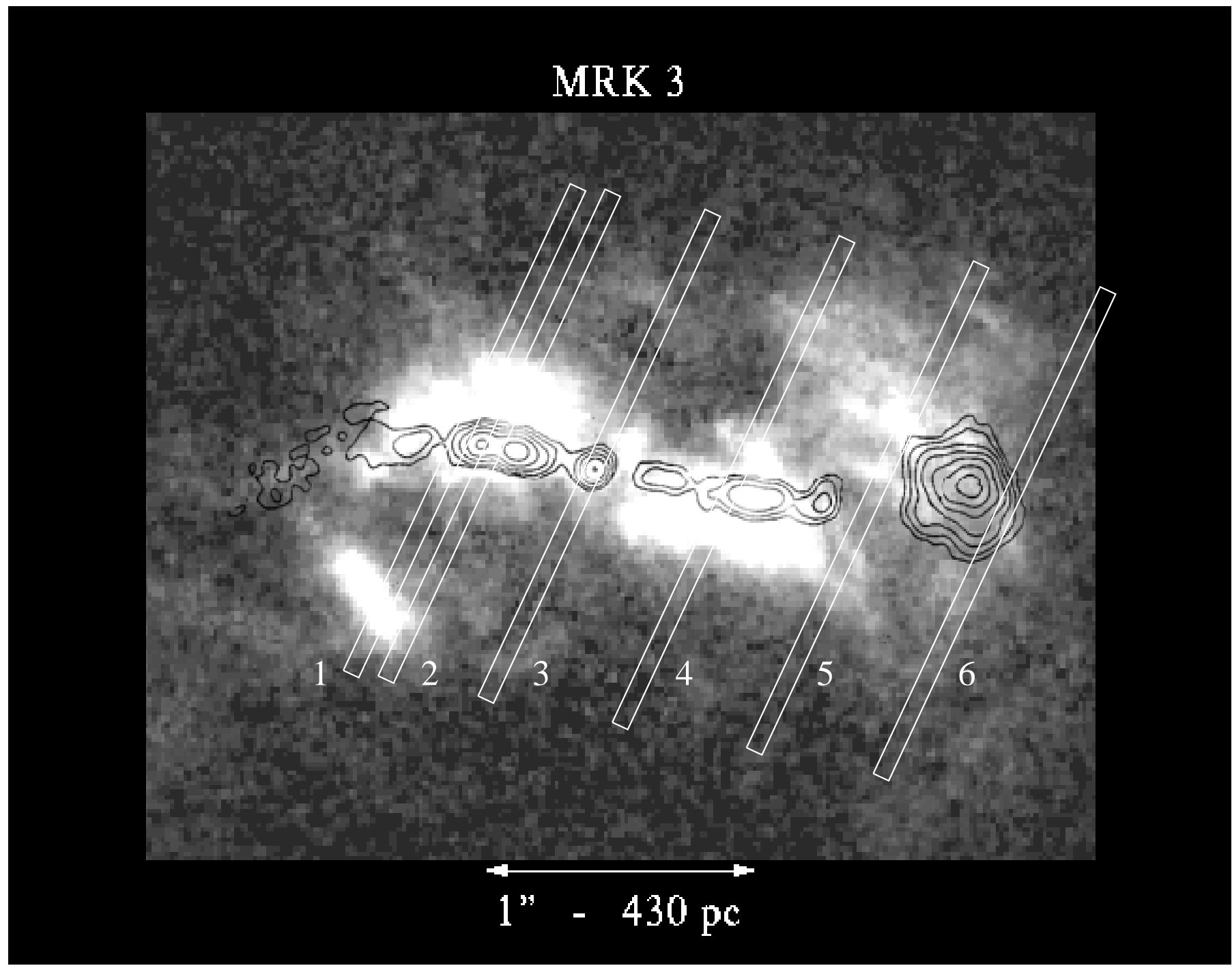

FIG. 1. - HST/FOC image of Mrk 3 in the [O III] emission line from Capetti et al. (1996); superposed are the contour radio image from Kukula et al. (1993) and the six slit positions at which the FOC $\mathrm{f} / 48$ spectra were taken. North is up and east is to the left.

radio ejecta (Wilson \& Willis 1980; Booler, Pedlar, \& Davis 1982; Pedlar et al. 1989; Taylor et al. 1989, 1992).

The Hubble Space Telescope (HST) allowed us for the first time to fully resolve the region from which the narrow emission lines originate. The results of extensive narrowband imaging of Seyfert galaxies clearly showed that the NLR morphology is completely determined by the presence of radio outflows (e.g., Bower et al. 1994, 1995; Capetti et al. 1995a, 1995b, 1996, 1997a, 1997b; Falcke et al. 1996, 1998). In particular, Seyfert galaxies with a lobelike radio morphology have bow-shock-shaped emission-line regions, while those with a jetlike radio structure have jetlike emission-line structures. These observations provide further compelling evidence for strong dynamical interactions between the NLR gas and radio-emitting ejecta.

In this framework, it is of great interest to study the effects of this interaction on the NLR dynamics at a high spatial resolution. This prompted a program of long-slit observations using the Faint Object Camera (FOC) in its spectrographic mode in order to study in detail the velocity field of the NLRs of Seyfert galaxies. The first results from observations of NGC 4151 (Winge et al. 1997, 1998) and NGC 1068 (Axon et al. 1998) show clear evidence that the highly perturbed and complex velocity field of the NLRs of these objects is strongly influenced by the jets' propagation. In particular, high-velocity gas components are associated univocally with the jet region.

We now present the results of long-slit $H S T / F O C$ spectroscopy of Mrk 3, a Seyfert 2 galaxy that presents one of the clearest examples of close association between radio and line emission. Radio images show a linear structure extending over $\sim 2$, , dominated by two symmetric and highly collimated jets (Kukula et al. 1993). HST emission-line images of Mrk 3 reveal that its NLR has a striking S-shaped morphology, composed of a series of knots, sheets, or filaments, and that it is basically cospatial with the radio jets (Capetti et al. 1995b, 1996).

In this paper, we show that the NLR of Mrk 3 is essentially an expanding shell surrounding the radio jet, whose velocity field is determined by the energy input of the outflowing jets. The detailed spatial and velocity information derived allow us to investigate the energetics of the jet/NLR 
interaction and to set constraints on the properties and evolution of the radio source associated with Mrk 3. We defer the discussion of line ratios and the ionization properties of the emitting gas to a forthcoming paper.

Throughout this paper, we adopt a recession velocity for Mrk 3 of $4000 \mathrm{~km} \mathrm{~s}^{-1}(z=0.0133)$, which, for $H_{0}=50 \mathrm{~km}$ $\mathrm{s}^{-1} \mathrm{Mpc}^{-1}$, yields a distance of $80 \mathrm{Mpc}$, where $1^{\prime \prime}$ corresponds to $390 \mathrm{pc}$.

\section{OBSERVATIONS AND DATA REDUCTION}

Mrk 3 was observed on 1996 December 9 using the FOC f/48 long-slit spectrograph. One pixel corresponds to $1.78 \AA$ and 0 ".0287 along the dispersion and slit directions, respectively. The F305LP filter was used to isolate the first-order spectrum, which covers the 3650-5470 ̊ region and therefore includes the [O II] $\lambda 3727, \mathrm{H} \beta \lambda 4861$, and [O III] $\lambda \lambda 4959,5007$ emission lines. An interactive-acquisition $1024 \times 512$ zoomed image was obtained with the $f / 48$ camera through the F342W filter to accurately locate the brightest line-emission regions. The slit, 0".063 × 13". 5 in size, was placed at a position angle of $-25^{\circ}$. Spectra with exposure times of $1000 \mathrm{~s}$ were taken in the $1024 \times 512$ nonzoomed mode at seven locations separated by 0 ". 4 . An additional spectrum of $780 \mathrm{~s}$ was taken immediately following the interactive acquisition image, before the telescope smallangle maneuver (position 2 in Fig. 1).

The data reduction follows the procedure described in detail by Macchetto et al. (1997). In summary, all frames, including those used for subsequent calibrations, were geometrically corrected by using the equally spaced grid of reseaux marks etched onto the first photocathode in the intensifier tube. Any remaining internal distortion was corrected by tracing the spectra of two stars in the core of the globular cluster 47 Tuc. The distortion along the spatial direction was obtained in a similar way, tracing the brightness distribution of the emission lines of the planetary nebula NGC 6543. Observations of NGC 6543 were also used to obtain the wavelength calibration. The instrumental broadening is estimated to be $\sim 320 \pm 20 \mathrm{~km} \mathrm{~s}^{-1}$. Flux calibration was performed using the observations of the spectrophotometric standard star LDS 749b.

In order to accurately determine the location of the slits, we compared the surface brightness profile derived from the HST FOC f/96 [O III] image of Capetti et al. (1996) with that measured from the spectra at the six slit positions that yielded useful data. The best match is displayed in Figure 1 and is accurate to within half a slit width $(\simeq 0$ " 03$)$. The slit locations, identified as POS1 through POS6, cut across the emission-line region of MRK 3 in a direction almost perpendicular to the radio axis, P.A. $+84^{\circ}$ (Kukula et al. 1993).

\section{RESULTS}

Velocities were derived by fitting Gaussian profiles to the [O III] $\lambda 5007$ line at each individual pixel using the task LONGSLIT in the TWODSPEC FIGARO package (Wilkins \& Axon 1992). In several locations the line is composed of more than one component, and in these cases multiple Gaussian fitting was performed. In regions of low signal-to-noise ratio, up to three pixels along the spatial direction were coadded. The resulting velocity profiles across the slit at each location are shown in Figures 2-8, where we also plot line widths and intensities. The origin of the $\mathrm{X}$-axis for each slit location is set at the intersection with the radio axis.

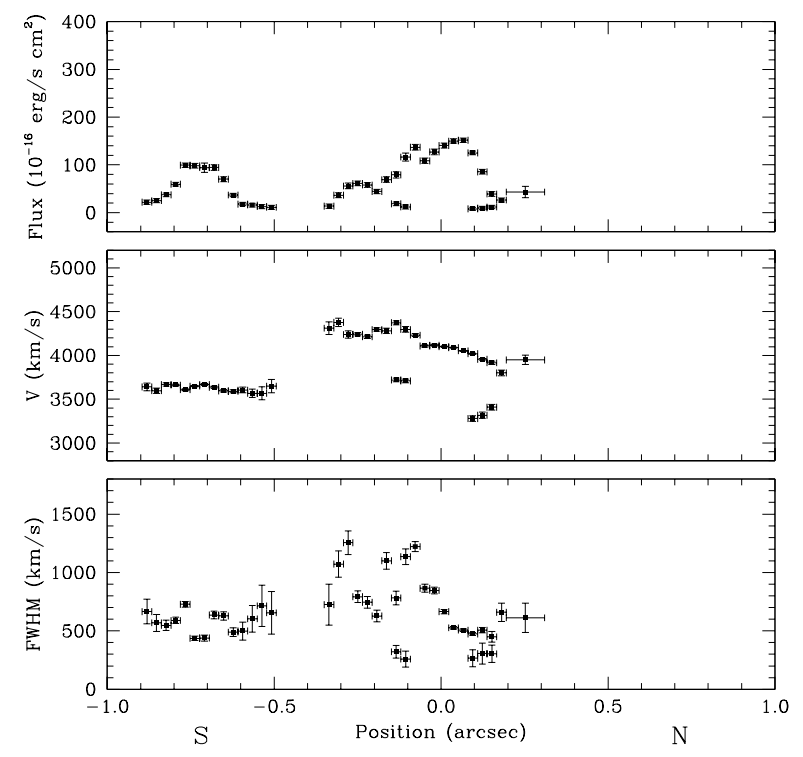

FIG. 2.-Intensity (top), velocity (middle), and line widths (bottom), measured at the slit position POS1.

\subsection{Description of the Individual Spectra}

POS1.-The line profiles are split into two distinct velocity systems in the region of the radio jet, with a separation of $\sim 1000 \mathrm{~km} \mathrm{~s}^{-1}$. Toward the south, only the redshifted component is visible. The region of high velocity is extended over $\sim 0.5$. The slit also crosses the bright southeastern blob. This blob is detached from the main NLR structure and is not directly associated with the radio jet. The gas in this region has a velocity of $\sim 3600 \mathrm{~km} \mathrm{~s}^{-1}$ and is significantly blueshifted from the velocity on the east side of the NLR, which is $\sim 4300 \mathrm{~km} \mathrm{~s}^{-1}$ (Wagner 1987; Metz 1998). Furthermore, the lines are quite broad, $\sim 700 \mathrm{~km} \mathrm{~s}^{-1}$.

POS2.-This slit position was obtained during the interactive acquisition and was located close to POS1. On the north side of the NLR, the velocity is approximately constant at around $3900 \mathrm{~km} \mathrm{~s}^{-1}$. Along the radio axis, there is a

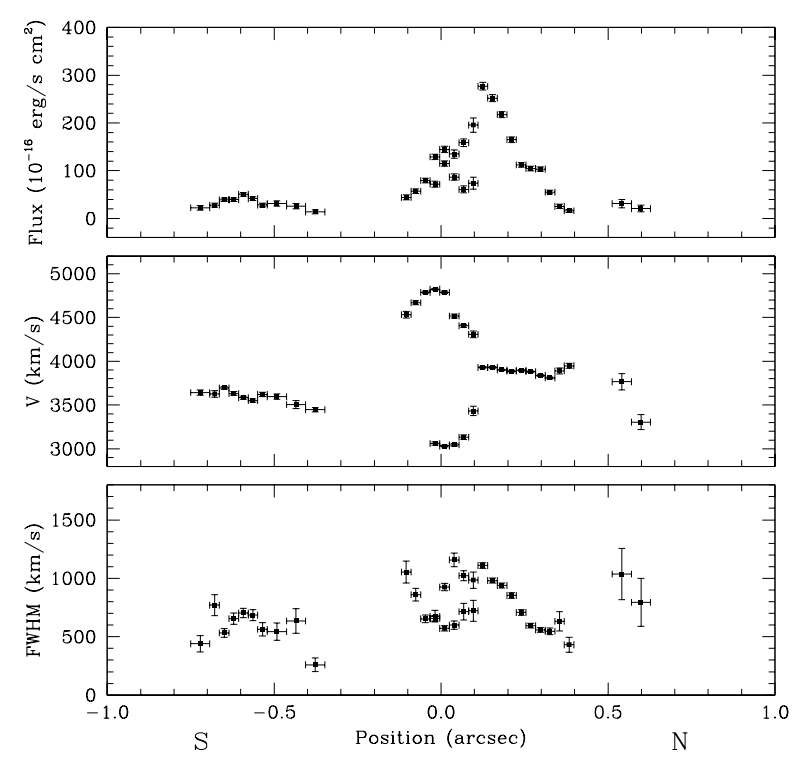

FIG. 3.-As in Fig. 2, for slit position POS2 


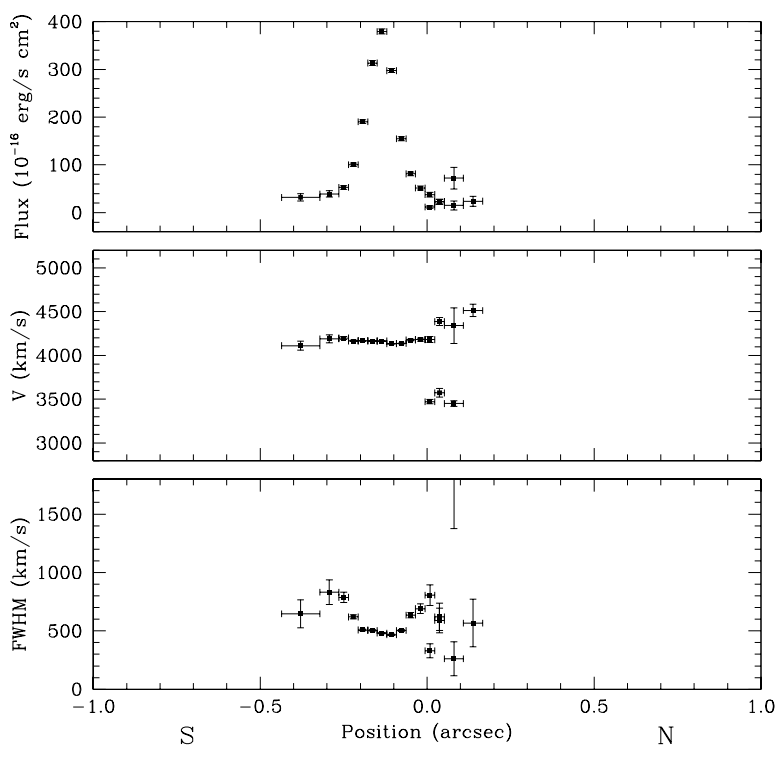

FIG. 4.- As in Fig. 2, for slit position POS3

dramatic split of the lines, with a separation that increases very rapidly and reaches a maximum of $1700 \mathrm{~km} \mathrm{~s}^{-1}$. Unlike in POS1, the velocity field forms a nearly complete velocity ellipsoid. We are seeing an expanding shell of size $\sim 0$."3 around the radio jet. The line widths of the individual components are always large, $500 \mathrm{~km} \mathrm{~s}^{-1}$, and significantly wider than the instrumental broadening of $320 \mathrm{~km} \mathrm{~s}^{-1}$. The large increase in line width just north of the split line region is probably caused by spatial confusion of the two velocity systems at the periphery of the shell. On the opposite side of the jet, the slit grazes the bright southeastern blob. A smooth gradient of $200 \mathrm{~km} \mathrm{~s}^{-1}$ can be seen over a distance of 0 . 4 , and again line widths are large, $\sim 700 \mathrm{~km} \mathrm{~s}^{-1}$.

POS3.- This slit is located on the brightest blob of the NLR of Mrk 3, close to its center of symmetry, and, most likely, to its hidden nucleus. The velocity field is remarkably flat on the blob, but the lines are characteristically broad

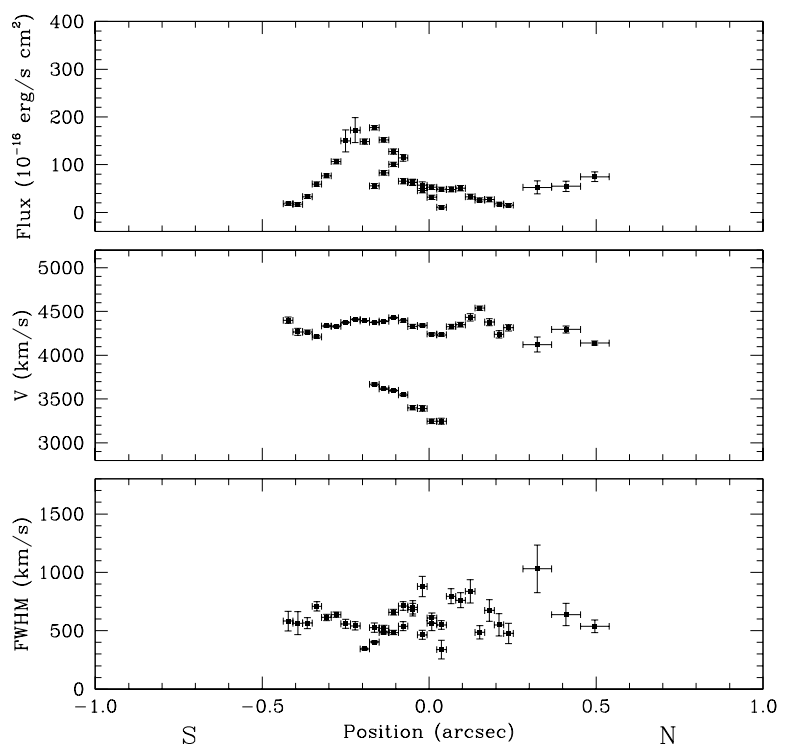

FIG. 5.-As in Fig. 2, for slit position POS4
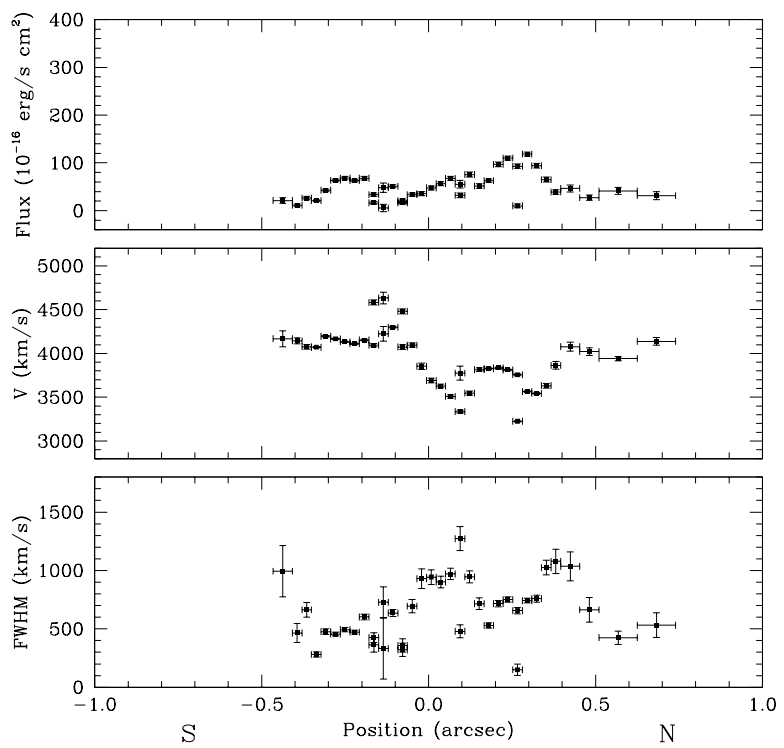

FIG. 6.-As in Fig. 2, for slit position POS5

$\left(500 \mathrm{~km} \mathrm{~s}^{-1}\right)$. The emission from this blob is redshifted by about $\sim 200 \mathrm{~km} \mathrm{~s}^{-1}$ with respect to the galaxy systemic velocity. Immediately north of this blob, corresponding to the point where the radio jet intersects the slit, lines are again split into two components, with a velocity separation of $\sim 1000 \mathrm{~km} \mathrm{~s}^{-1}$.

POS4.-This location is symmetric to POS1 with respect to the nucleus, and the velocity field is very similar, but with a reversed symmetry. Again, it shows a very large line splitting $\left(1200 \mathrm{~km} \mathrm{~s}^{-1}\right)$ along the radio jet over $\sim 0$ ".4, in the form of a broken velocity ellipsoid.

POS5.-The gas kinematics here are complex. Three highly perturbed regions correspond to three bright filaments crossed by the slit. The first, toward the south, is part of the main NLR structure. The remaining two, which are blueshifted by $\sim 600 \mathrm{~km} \mathrm{~s}^{-1}$ and have large line widths, are associated with the radio lobe. These structures are super-

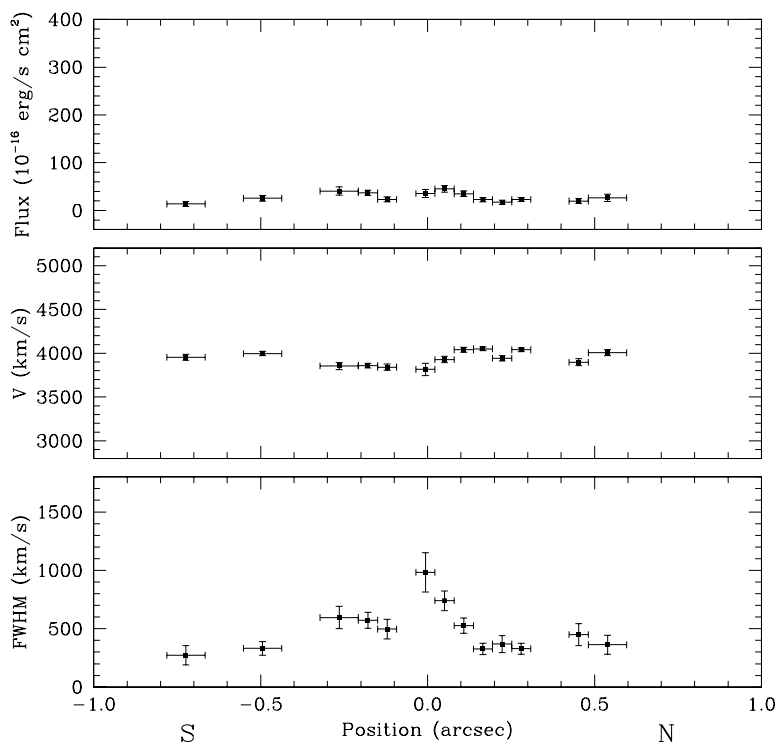

FIG. 7.-As in Fig. 2, for slit position POS6 


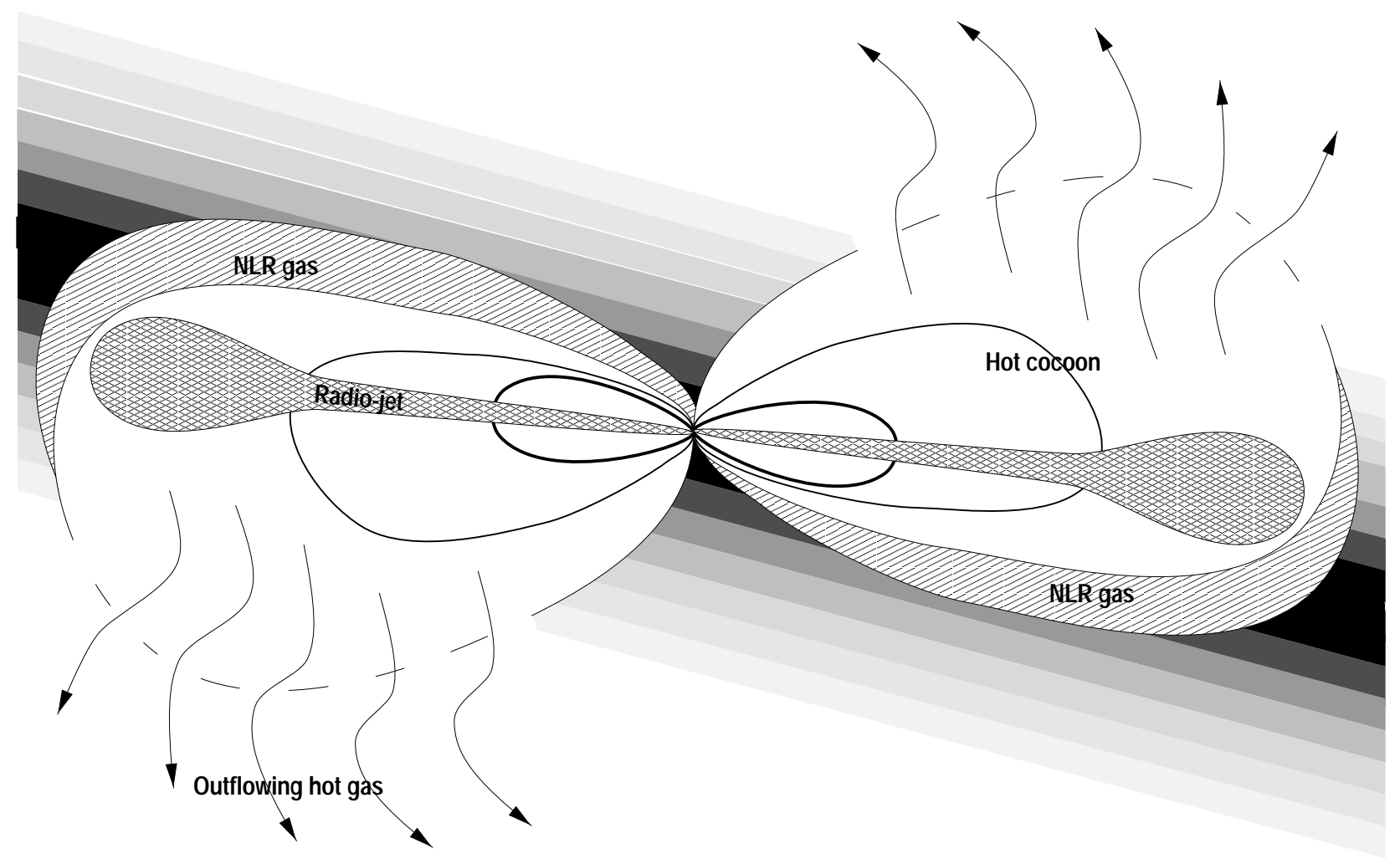

Hot cocoon

FIG. 8.-Cartoon illustrating the effects of the relative jet/disk orientation on the cocoon expansion and on the NLR morphology

posed onto a more regular pattern, determined by the diffuse emission. Such complex line profiles have been seen previously on the lobe of the powerful radio Seyfert galaxy IRAS $04210+0400$ (Holloway et al. 1996).

POS6.-Slit location 6 grazes the edges of the NLR and of the western radio lobe in a region of diffuse line emission. While the velocity field is essentially quiescent, with a dispersion of less than $250 \mathrm{~km} \mathrm{~s}^{-1}$, the line profiles are significantly broad only in the region along the radio axis, with a FWHM as high as $1000 \mathrm{~km} \mathrm{~s}^{-1}$.

\section{KINEMATICS OF THE NLR GAS}

The most dramatic result is that, kinematically, the NLR of Mrk 3 takes the form of a broken velocity ellipsoid centered on the radio jet. This is the expected behavior of an expanding cocoon of gas circumscribing the radio jet, and is unambiguous evidence that the NLR is created by the interaction of the radio jet with the interstellar medium. Note that while there is a misalignment between the NLR and radio axis (Capetti et al. 1995b), the velocity ellipsoid is centered firmly on the jet. Line widths in the expanding regions are always broad $\left(500 \mathrm{~km} \mathrm{~s}^{-1}\right)$, suggesting significant turbulence within the cocoon.

The diameter of the emission-line shell ( $\sim 200 \mathrm{pc})$ is much larger than the radio jet, which is unresolved in the MERLIN images $(d<15 \mathrm{pc})$. This is an indication that the radio plasma does not interact directly with the lineemitting gas. This is physically expected, since for a shock velocity of $\gtrsim 1000 \mathrm{~km} \mathrm{~s}^{-1}$, the postshock temperature will be of the order of $\gtrsim 10^{7} \mathrm{~K}$ (Taylor et al. 1992). A hot highpressure cocoon then develops around the advancing radio jet and mediates the energy exchange between jets and lineemitting gas. At these temperatures, the main cooling mechanism is free-free radiation rather than line emission. Strong support for this interpretation is the direct detection of the ultraviolet counterpart to the Mrk 3 jet (Axon et al. 1999).

The excess pressure of the hot gas initially drives the supersonic NLR expansion. However, we believe that the NLR is currently in a momentum-driven phase, since the bubble has been punctured. The two key facts leading to this conclusion are the mirror symmetry about the nucleus of the NLR emissivity and fractures in the velocity ellipsoids. As described above, on the western jet the bubble is open toward the south and the peak of emission is to the north (POS1 and POS2), while the reverse is seen along the eastern jet (POS3 through POS5). This behavior can be explained if the jets are propagating in the stratified gas of a disk galaxy and the jets are inclined with respect to the gas disk (see Fig. 8). The dynamical evolution is very similar to that of the wind-driven superbubbles associated with OB associations or supernovae in the ISM (Tomisaka \& Ikeuchi 1986; Mac Low \& McCray 1988). Initially, the bubble will expand asymmetrically with respect to the jet axis, with the side away from the disk expanding more rapidly. It will still appear as a closed, although asymmetric, velocity ellipsoid. The bright emission line region corresponds to the side of the bubble that is snow-plowing into the disk. At this stage, the NLR motions are pressure driven. When the bubble grows to be large enough, it is more profoundly influenced by the stratification of the galactic disk. With its current size of $200 \mathrm{pc}$, the cocoon has 
probably expanded to several disk scale heights, and the hot gas located above the plane of the disk is blowing out into the halo, puncturing the bubble. At this time the system is effectively momentum driven.

In addition to the highly perturbed gas on which we have concentrated so far, at all slit locations there are extended regions in which the gas is relatively quiescent, i.e., with a velocity field characterized by small velocity gradients, small offset from the galaxy systemic velocity, and relatively narrow line profiles. This gas is probably associated with the galaxy disk. The presence of an extended rotating gas disk in Mrk 3 is clearly seen in ground-based data (Metz 1998), and it is normally observed in Seyfert galaxies. A similar two-components gas structure in the NLR has recently also been seen in NGC 4151 (Winge et al. 1998). Radio outflows only locally disrupt the ordered rotation pattern.

\section{EVOLUTION OF THE COCOON}

The evolution of an overpressured cocoon driven by a supersonic jet has been studied by several authors in the framework of powerful radio galaxies. The same approach can be applied to the expanding cocoon in Mrk 3, but in this case, we can directly measure its volume and expansion velocity, which turn out to be crucial parameters for constraining the model.

Begelman \& Cioffi (1989) showed that the evolution of a radio source is determined by the balance between the ram pressure of the external medium and the thrust of the jet, and (where the lateral expansion is concerned) the cocoon internal pressure, i.e., $p_{c} \sim \rho_{a} v_{c 0}^{2}$, where $p_{c}$ and $v_{c 0}$ are the cocoon pressure and the present expansion velocity, respectively, and $\rho_{a}$ is the external density. The medium in which the jet propagates is likely to be clumpy, and the evolution of a radio source within a nonuniform medium greatly resembles the way in which it would interact with a smooth medium with the same average density (De Young 1993). In this situation, the relevant value for $\rho_{a}$ is the density averaged over the volume of the radio source.

The cocoon internal pressure is maintained by the energy carried by the jet. For a jet power of $L_{j}$, averaged over the radio-source lifetime $t_{\mathrm{rs}}$, we have $p_{c} \sim 2 t_{\mathrm{rs}} L_{j} / V_{c}$. The cocoon volume, $V_{c}$, is given by $V_{c} \sim 2 d_{c}^{2} l_{c}$, where $d_{c}$ and $l_{c}$ are its diameter and length, respectively.

The source age can be estimated by noting that in such a cylindrical symmetry the expansion speed decreases as $t^{-1 / 2}$, i.e., $v_{c}=v_{c 0}\left(t / t_{\mathrm{rs}}\right)^{-1 / 2}$. Integrating the cocoon expansion from $t=0$ to $t=t_{\mathrm{rs}}$, we get

$$
\begin{aligned}
d_{c} & \sim 2 \int_{0}^{t_{\mathrm{rs}}}\left(t / t_{\mathrm{rs}}\right)^{-1 / 2} v_{c 0} d t \sim 4 v_{c 0} t_{\mathrm{rs}}, \\
t_{\mathrm{rs}} & \sim \frac{d_{c}}{4 v_{c 0}} .
\end{aligned}
$$

We can now express the jet power as

$$
L_{j} \sim \rho_{a} v_{c 0}^{2} d_{c}^{2} l_{c} t_{\mathrm{rs}}^{-1} \sim 4 \rho_{a} v_{c 0}^{3} d_{c} l_{c} .
$$

Except for the external density, $\rho_{a}$, the above parameters can be directly measured from our imaging and spectroscopy data of Mrk 3 . We have $d_{c} \sim 200 \mathrm{pc}, l_{c} \sim 500 \mathrm{pc}$, and $v_{c 0} \sim 700 \mathrm{~km} \mathrm{~s}^{-1}$, from which we get a jet power of

$$
L_{j} \sim 2 \times 10^{42} \rho_{1} \operatorname{ergs~s}^{-1},
$$

where $\rho_{1}$ is the average density of the unperturbed gas in units of one particle per cubic centimeter, a value that is appropriate to the interstellar medium of our Galaxy (Cox \& Reynolds 1987).

As for the radio-source age, the value that corresponds to the expansion law we adopted is $t_{\mathrm{rs}} \sim 7 \times 10^{4} \mathrm{yr}$. In reality, the cocoon lateral evolution is very complex and depends on the precise distribution of the external medium and mass loading in the shell. The issue of mass loading of astronomical flows has been discussed extensively in the literature (e.g., Hartquist et al. 1986), and it will be important in the evolution of the cocoon around the jet of Mrk 3. In addition, the bubble has now been punctured, and it is in a momentum-driven phase. A firm upper limit of $1.5 \times 10^{5} \mathrm{yr}$ on the radio-source dynamical timescale can be set by assuming a constant expansion at the present speed. On the other hand, a lower bound to the age of the outflow can be set if one assumes that the ionizing photons switch on at the same time as the radio ejecta. The size of the extended narrow-line region of Mrk 3 ( $\sim 4 \mathrm{kpc}$; Pogge \& De Robertis 1993) sets it at $\sim 10^{4}$ yr. This would correspond to an expansion speed of less than $0.1 c$, similar to what is derived for powerful radio galaxies.

If the NLR dynamics are indeed dominated by the cocoon expansion, the jet must have carried enough energy to accelerated the emitting gas. We can estimate the total kinetic energy of the high-velocity gas, operatively defined as the gas within 0 .'2 from the radio jet with a velocity that differs by more than $300 \mathrm{~km} \mathrm{~s}^{-1}$ from the systemic velocity. A given $\mathrm{H} \beta$ luminosity of $L_{\mathrm{H} \beta}$ corresponds to a mass of ionized gas (Osterbrock 1989)

$$
M_{\mathrm{gas}} \sim 7.5 \times 10^{-3} \frac{10^{4}}{n_{e}} \frac{L_{\mathrm{H} \beta}}{L_{\odot}} M_{\odot} .
$$

Adopting an average $[\mathrm{O} \mathrm{III}] / \mathrm{H} \beta$ ratio of 12.6 and an average density of $700 \mathrm{~cm}^{-3}$ (Metz 1998), an observed flux in the [O III] line of $10^{-16} \mathrm{ergs} \mathrm{s}^{-1} \mathrm{~cm}^{-2}$ corresponds to a mass of $\sim 130 M_{\odot}$. Integrating over the region of interest, we find a total [O III] flux of $4.3 \times 10^{-13} \mathrm{ergs} \mathrm{s}^{-1} \mathrm{~cm}^{-2}$ (about $20 \%$ of the total NLR emission) and a kinetic energy of $1.2 \times 10^{54}$ ergs. Since only about $1 / 5$ of the jet length is covered by our slits, the total kinetic energy of the gas associated with the radio jet is $\sim 6 \times 10^{54}$ ergs. On the other hand, during the radio-source expansion, half of the energy pumped by the jet goes into the cocoon's internal energy, while the other half goes into accelerating the external medium. This strictly applies only if the radiative losses of the cocoon are negligible. This is indeed the case, since the cooling time for a temperature of $10^{7} \mathrm{~K}$ is $\sim 1.5 \times 10^{6}$ $\rho^{-1} \mathrm{yr}$, where $\rho$ is the gas density, much longer than the radio-source dynamical time. The total energy deposited by the jet over the radio-source lifetime is $\sim 5 \times 10^{54}$ ergs, remarkably similar to the kinetic energy of the line-emitting gas. This provides quantitative support for the idea that the gas is accelerated (indirectly) by the jet.

\section{DISCUSSION}

Radio outflows are known to be commonly associated with Seyfert galaxies; in at least $50 \%$ of the cases, the radio emission is extended, and it often shows a linear structure (Ulvestad \& Wilson 1989; Kukula et al. 1995), with typical sizes of less than $\sim 1 \mathrm{kpc}$, rarely extending beyond $3 \mathrm{kpc}$. For Mrk 3, we estimated that the radio source size has 
increased at a minimum speed of $\sim 3500 \mathrm{~km} \mathrm{~s}^{-1}\left(3 \times 10^{-3}\right.$ pc or $8 \mu$ as per year). Recently, $H S T / F O C$ spectroscopy has also been obtained for NGC 1068 and NGC 4151 (Axon et al. 1998; Winge et al. 1997). The lateral expansion of the NLR gas in these two objects occurs at a speed similar to that in Mrk 3. In particular, NGC 1068 shows a welldeveloped jet-cocoon structure, whose transverse size is $\sim 100$ pc. The radio-source sizes of NGC 1068 and NGC 4151 are also comparable to that of Mrk $3(900$ and $600 \mathrm{pc}$, respectively; Wilson \& Ulvestad 1987; Pedlar et al. 1993); we therefore naturally get dynamical timescales similar to that of Mrk 3. This suggests that the rate of linear size expansion of the radio source of Mrk 3 is not unusually high, but is representative of Seyfert galaxies with linear radio structures. If this is the case, the lifetimes of radio outflows in Seyfert galaxies must be typically $\lesssim 10^{5} \mathrm{yr}$; otherwise, we should observe Seyfert radio sources extending well beyond $1 \mathrm{kpc}$. As a comparison, the source age for radio galaxies ranges from $10^{6}$ to $10^{8} \mathrm{yr}$ (Alexander \& Leahy 1987; Liu, Pooley, \& Riley 1992; Parma et al. 1998). The longer timescales associated with radio galaxies are also confirmed by the discovery of a line-emitting expanding shell surrounding the jet of $3 \mathrm{C} 120$, which points to an age for this source of $6 \times 10^{6} \mathrm{yr}$ (Axon et al. 1989). It appears that the phase of radio activity in Seyfert galaxies is relatively short lived.

Two alternative scenarios are viable at this stage. The high nuclear luminosity and the radio activity may be causally related and thus last over similar timescales, in which case the whole Seyfert phenomenon will be short lived. The other possibility is that the radio activity in Seyfert galaxies is recurrent.

We can compare the radio, optical, and jet luminosities of Mrk 3 with those of other classes of active galactic nuclei. Its [O III] luminosity is $L_{[\mathrm{O}} \mathrm{m]}=2.1 \times 10^{42} \mathrm{ergs} \mathrm{s}^{-1}$ (Koski 1978), while the radio luminosity is $L_{r}=2.7 \times 10^{30} \mathrm{ergs} \mathrm{s}^{-1}$ $\mathrm{Hz}^{-1}$ at $4.8 \mathrm{GHz}$ (Ulvestad \& Wilson 1984; Becker, White, \& Edwards 1991) and $L_{r}=2.4 \times 10^{31} \mathrm{ergs} \mathrm{s}^{-1} \mathrm{~Hz}^{-1}$ at 178 $\mathrm{MHz}$ (Gower, Scott, \& Wills 1967). The integrated radio spectral index is then 0.66 , from which we derive a radio luminosity for Mrk 3 between $10 \mathrm{MHz}$ and $100 \mathrm{GHz}$ of $\sim 10^{41} \mathrm{ergs} \mathrm{s}^{-1}$.

The point representing Mrk 3 in the radio/optical luminosity plane falls within the region defined by the radioquiet (RQ) quasar sample at $z<0.5$ studied by Miller, Rawlings, \& Saunders (1993), which covers the line luminosity range of $10^{41}-10^{44} \mathrm{ergs} \mathrm{s}^{-1}$. Conversely, the radio luminosity, $L_{r}$, of Mrk 3 is between 2 and 3 orders of magnitude below the average of the radio-loud (RL) active galactic nuclei (AGNs) with similar line luminosities, $L_{\mathrm{NLR}}$ (Rawlings et al. 1989). The Mrk 3 jet power, $L_{j}$ (estimated from the expansion velocity of the emission-line region associated with the radio jet), can be compared with estimates based on the equipartition parameters (Rawlings \& Saunders 1991) for RL AGNs. Mrk 3 again lies between 2 and 3 orders of magnitude below the region defined by the objects having similar values of $L_{\mathrm{NLR}}$. On the other hand, the ratio between $L_{j}$ and $L_{r}$ is similar for Mrk 3 and RL AGNs, indicating that a similar fraction of jet power is dissipated in radio emission. More quantitatively, the efficiency at which the jet power is converted into radio emission in Mrk 3 is $0.05 \rho_{1}$. We speculate that the low radio luminosity of RQ objects is due to the presence of intrinsically less energetic outflows than in RL objects, rather than to a lower efficiency in producing synchrotron emission. Clearly, observations of a sample of radio-quiet active nuclei with nuclear outflows are required before firm conclusions can be drawn.

\section{SUMMARY AND CONCLUSIONS}

Our HST/FOC long-slit spectroscopic observations clearly show that the kinematics of the gas in the NLR of Mrk 3 are determined by the effects of the interaction between the radio outflow and the ambient gas. In particular, along the radio jet the line-emitting gas shows two velocity systems separated by as much as $1700 \mathrm{~km} \mathrm{~s}^{-1}$, which form broken velocity ellipsoids. This is a clear indication that the gas is expanding away from the radio-jet axis and that the NLR is essentially a cylindrical shell expanding supersonically. These results are in close agreement with what we found in the NLRs of other Seyfert galaxies observed with $H S T$.

The physical picture that emerges is that the highvelocity shocks induced by the outflowing plasma are heating and compressing the external gas. Close to the radio jets, the gas is heated to a high temperature, $10^{7} \mathrm{~K}$, thus forming a hot cocoon surrounding the jets. While its emission (mostly in the X-ray) can be relevant for the ionization properties of the NLR, it is negligible for the overall energy budget of the radio source; thus, the cocoon will undergo an essentially adiabatic expansion. During this expansion it acts as a piston, accelerating and compressing the surrounding gas that is responsible for the narrow-line emission. The jet kinetic energy is therefore transferred to the line-emitting gas after it is dissipated in thermal energy associated with the cocoon.

With its current size of $200 \mathrm{pc}$, the cocoon has expanded to several disk scale heights. The hot gas located above the plane of the disk is probably escaping into the halo, puncturing the bubble as indicated by the fractures in the velocity field. We believe that the NLR is currently in a momentum-driven phase.

In addition to the highly perturbed gas associated with the radio jet, a relatively quiescent component is also present, probably associated with the galaxy disk. The radio outflow only locally disrupts its ordered rotation pattern.

In the framework in which the gas motions are driven by the radio jets, it is possible to derive several key physical parameters describing the properties of the radio source associated with Mrk 3. In particular, from the size of the cocoon and its lateral expansion speed, we estimated an upper limit to its age $\left(\lesssim 1.5 \times 10^{5} \mathrm{yr}\right)$ and a lower limit to the jet kinetic power $\left(\gtrsim 2 \times 10^{42}\right.$ ergs s$\left.^{-1}\right)$. This provides quantitative support for the proposed NLR model, since the total energy carried by the jet in its lifetime is approximately equal to the kinetic energy associated with the perturbed high-velocity NLR gas, $\sim 6 \times 10^{54}$ ergs.

Furthermore, if the advance speed of the Mrk 3 radio source is taken to be representative, it implies that radio outflows associated with Seyfert galaxies are short lived $\left(\lesssim 10^{5} \mathrm{yr}\right)$, since their typical sizes are smaller than a few kpc.

Finally, while the jet kinetic luminosity of Mrk 3 is between 2 and 3 orders of magnitude smaller than that derived for RL AGNs with similar emission-line luminosities, the fraction of the jet power dissipated in radio emission is similar to that of RL AGNs. We thus speculate that the main distinction between RQ and RL AGNs is to 
be ascribed to a different jet power rather than to a different efficiency in producing synchrotron emission.

Exploring the effects of the jet propagation within the NLRs of active nuclei is clearly a very promising tool for studying the jet physics and for relating the properties of different classes of AGNs. In particular, it will be important to study the dynamical timescales for a statistically significant number of Seyfert and radio galaxies to further test the proposed model.
Alexander, P., \& Leahy, J. P. 1987, MNRAS, 225, 1

Axon, D. J., Capetti, A., \& Macchetto, F. D. 1999, ApJ, submitted

Axon, D. J., Marconi, A., Capetti, A., Macchetto, F. D., Schreier, E. A., \& Robinson, A. 1998, ApJ, 496, L75

Axon, D. J., Pedlar, A., Unger, S. W., Meors, E. J. A., \& Whittle, D. M. 1989, Nature, 341, 631

Baldwin, J. A., Wilson, A. S., \& Whittle, M. 1987, ApJ, 319, 84

Becker, R. H., White, R. L., \& Edwards, A. L. 1991, ApJS, 75, 1

Begelman, M., \& Cioffi, D. F. 1989, ApJ, 345, L21

Booler, R. V., Pedlar, A., \& Davies, R. D. 1982, MNRAS, 199, 229

Bower, G., Wilson, A. S., Morse, J. A., Gelderman, R., Whittle, M., \& Mulchaey, J. 1995, ApJ, 454, 106

Bower, G., Wilson, A. S., Mulchaey, J., Miley, G. K., Heckman, T. M., \& Krolik, J. H. 1994, AJ, 107, 1686

Capetti, A., Axon, D. J., Kukula, M., Macchetto, F., Pedlar, A., Sparks, W. B. \& Boksenberg, A. 1995a, ApJ, 454, L85

Capetti, A., Axon, D. J., \& Macchetto, F. D. 1997a, ApJ, 487, 560

Capetti, A., Axon, D. J., Macchetto, F. D., Sparks, W. B., \& Boksenberg, A. 1996, ApJ, 469, 554

Capetti, A., Macchetto, F. D., \& Lattanzi, M. G. 1997b, ApJ, 476, L67

Capetti, A., Macchetto, F. D., Sparks, W. B., \& Boksenberg, A. 1995b, ApJ, 448,600

Cox, D. P., \& Reynolds, R. J. 1987, ARA\&A, 25, 303

De Young, D. S. 1993, ApJ, 402, 95

Falcke, H., Wilson, A. S., \& Simpson, C. 1996, ApJ, 470, L31 1998, ApJ, 502, 199

Gower, J. F. R., Scott, P. L., \& Wills, D. 1967, MmRAS, 71, 49

Haniff, C. A., Wilson, A. S., \& Ward, M. J. 1988, ApJ, 334, 104

Hartquist, T. W., Dyson, J. E., Pettini, M., \& Smith, L. J. 1986, MNRAS, 221,715

Holloway, A. J., Steffen, W., Pedlar, A., Axon, D. J., Dyson, J. E., Meaburn, J., \& Tadhunter, C. N. 1996, MNRAS, 279, 171

Koski, A. T. 1978, ApJ, 223, 56

Kukula, M. J., Ghosh, T., Pedlar, A., Schilizzi, R. T., Miley, G. K., de Bruyn, A. G., \& Saikia, D. J. 1993, MNRAS, 264, 893

Kukula, M. J., Pedlar, A., Baum, S. A., \& O’Dea, C. P. 1995, MNRAS, 276, 1262

\section{EFERENCES}

Liu, R., Pooley, G. G., \& Riley, J. M. 1992, MNRAS, 257, 545

Macchetto, F. D., Marconi, A., Axon, D. J., Capetti, A., Sparks, W. B., \& Crane, P. 1997, ApJ, 489, 579

Mac Low, M.-M., \& McCray, R. 1988, ApJ, 324, 776

Metz, S. K. 1998, Ph.D. thesis, Univ. Hertfordshire (U.K.)

Miller, P., Rawlings, S., \& Saunders, R. 1993, MNRAS, 263, 425

Osterbrock, D. E. 1989, Astrophysics of Gaseous Nebulae \& Active Galactic Nuclei (Mill Valley: University Science Books)

Parma, P., Murgia, M., Morganti R., Capetti, A., de Ruiter, H. R., \& Fanti, R. 1998, A\&A, submitted

Pedlar, A., Meaburn, J., Axon, D. J., Unger, S. W., Whittle, D. M., Meurs, E. J. A., Guerrine, N., \& Ward, M. J. 1989, MNRAS, 238, 863

Pedlar, A., et al. 1993, MNRAS, 263, 471

Pogge, R. W., \& De Robertis, M. M. 1993, ApJ, 404, 563

Rawling, S., \& Sanders, R. 1991, Nature, 349, 138

Rawlings, P., Saunders, R., Eales, S. A., \& MacKay, C. D. 1989, MNRAS, 240,701

Taylor, D., Dyson, J. E., \& Axon, D. J. 1992, MNRAS, 255, 351

Taylor, D., Dyson, J. E., Axon, D. J., \& Pedlar, A. 1989, MNRAS, 240, 487

Tomisaka, K., \& Ikeuchi, S. 1986, PASJ, 38, 697

Ulvestad, J. S., \& Wilson, A. S. 1984, ApJ, 278, 544 . 1989, ApJ, 343, 659

Wagner, S. J. 1987, A\&A, 185, 77

Whittle, M. 1985, MNRAS, 216, 817

Whittle, M., Pedlar, A., Meurs, E. J. A., Unger, S. W., Axon, D. J., \& Ward, M. J. 1988, ApJ, 326, 125

Wilkins, T. W., \& Axon, D. J. 1992, in ASP Conf. Ser. 25, Astronomical Data Analysis Software and Systems I, ed. D. M. Worrall, C. Biemesderfer, \& J. Barnes (San Francisco: ASP), 427

Wilson, A. S., \& Ulvestad, J. S. 1983, ApJ, 275, 8 1987, ApJ, 319, 105

Wilson, A. S., \& Willis, A. G. 1980, ApJ, 240, 429

Winge, C., Axon, D. J., Macchetto, F. D., \& Capetti, A. 1997, ApJ, 487, L121

Winge, C., Axon, D. J., Macchetto, F. D., Capetti, A., \& Marconi, A. 1998, ApJ, submitted 